\title{
Study of Chemical Etching and Chemo-Mechanical Polishing on CdZnTe Nuclear Detectors
}

\author{
Aaron L. Adams ${ }^{1,2}$, Stephen U. Egarievwe, $2,3,4$ (D), Ezekiel O. Agbalagba5, Rubi Gul2,3, \\ Anwar Hossain 3 , Utpal N. Roy ${ }^{3}$, Ralph B. James ${ }^{6}$ \\ ${ }^{1}$ Department of Mechanical \& Civil Engineering, and Construction Management, Alabama A\&M University, \\ Huntsville, AL, USA \\ ${ }^{2}$ Nuclear Engineering and Radiological Science Center, Alabama A\&M University, Huntsville, AL, USA \\ ${ }^{3}$ Department of Nonproliferation and National Security, Brookhaven National Laboratory, Upton, NY, USA \\ ${ }^{4}$ Department of Electrical Engineering and Computer Science, Alabama A\&M University, Huntsville, AL, USA \\ ${ }^{5}$ Department of Physics, Federal University of Petroleum Resources, Effurun, Nigeria \\ ${ }^{6}$ Science and Technology, Savannah River National Laboratory, Aiken, SC, USA \\ Email: aaron.adams@aamu.edu
}

How to cite this paper: Adams, A.L., Egarievwe, S.U., Agbalagba, E.O., Gul, R., Hossain, A., Roy, U.N. and James, R.B. (2019) Study of Chemical Etching and Chemo-Mechanical Polishing on CdZnTe Nuclear Detectors. Journal of Materials Science and Chemical Engineering, 7, 33-41. https://doi.org/10.4236/msce.2019.78005

Received: June 25, 2019

Accepted: August 10, 2019

Published: August 13, 2019

Copyright (c) 2019 by author(s) and Scientific Research Publishing Inc. This work is licensed under the Creative Commons Attribution International License (CC BY 4.0).

http://creativecommons.org/licenses/by/4.0/

\section{cc) (i) Open Access}

\begin{abstract}
Cadmium zinc telluride (CdZnTe) semiconductor has applications in the detection of X-rays and gamma-rays at room temperature without having to use a cooling system. Chemical etching and chemo-mechanical polishing are processes used to smoothen CdZnTe wafer during detector device fabrication. These processes reduce surface damages left after polishing the wafers. In this paper, we compare the effects of etching and chemo-mechanical polishing on CdZnTe nuclear detectors, using a solution of hydrogen bromide in hydrogen peroxide and ethylene glycol mixture. X-ray photoelectron spectroscopy (XPS) was used to monitor $\mathrm{TeO}_{2}$ on the wafer surfaces. Currentvoltage and detector-response measurements were made to study the electrical properties and energy resolution. XPS results showed that the chemical etching process resulted in the formation of more $\mathrm{TeO}_{2}$ on the detector surfaces compared to chemo-mechanical polishing. The electrical resistivity of the detector is of the order of $10^{10} \Omega-\mathrm{cm}$. The chemo-mechanical polishing process increased the leakage current more that chemical etching. For freshly treated surfaces, the etching process is more detrimental to the energy resolution compared to chemo-mechanically polishing.
\end{abstract}

\section{Keywords}

CdZnTe, Chemical Etching, Chemo-Mechanical Polishing, Gamma Rays, 
Nuclear Detectors, X-Ray Photoelectron Spectroscopy

\section{Introduction}

The ability to operate at room temperature without cryogenic cooling has made cadmium zinc telluride ( $\mathrm{CdZnTe}$ ) nuclear detectors popular in X-ray detection and gamma-ray spectroscopy applications [1] [2] [3] [4] [5]. The absence of the need to cool the detector gives the advantage of reduced operational costs and the production of hand-held devices, when compared to detectors that need cooling, such as germanium-based detectors. The major application areas for $\mathrm{CdZnTe}$ detectors include national security, environmental protection.

Imaging, and astrophysics [6] [7] [8]. The fabrication of CdZnTe detectors involve cutting wafers from the ingot, mechanically polishing the wafers, chemical cleaning and treatment, and deposition of electrical contacts. The chemical cleaning process could be etching or chemo-mechanical polishing. These processes are used to remove surface defects that are left after the mechanical polishing of the wafers. The chemical treatment process involves passivation to make the wafer surfaces more chemically stable [4] [9] [10] [11].

While there have been many studies on the chemical treatment of CdZnTe detector, very little has been done on using the same chemical to compare the processes of etching and chemo-mechanical polishing [12]. In a recent study [12], we compared the effects of chemical etching and chemo-mechanical polishing on the electrical properties of $\mathrm{CdZnTe}$ detectors using bromine-methanol-ethylene glycol. The chemo-mechanical polishing process was observed to contribute lesser surface current to the measured current while the chemical etching process gave a higher charge-carrier mobility-lifetime product and radiation detection energy resolution [12]. The new investigation in the present study covers the effects on the tellurium and tellurium-oxide species on the surface of the CdZnTe wafers. The major differences between the previous study [12] and the present investigation include the chemical used, and the measurements of Te and $\mathrm{TeO}_{2}$ species on the surfaces of the wafers. The previous study used bromine-methanol-ethylene glycol [12]. In the present work, we used a solution of hydrogen bromide in hydrogen peroxide and ethylene glycol mixture. The Te and $\mathrm{TeO}_{2}$ species on the surfaces of the wafers were measured using X-ray Photoelectron Spectroscopy (XPS).

\section{Experiments}

\subsection{Preparation of Samples}

The CdZnTe used in this study was obtained from eV Products. It was grown by the vertical Bridgman method [13] [14] [15]. A special cutting machine equipped with a diamond wire saw was used to cut two samples (Sample-1 and Sample-2) from a larger CdZnTe wafer. The samples were polished mechanically 
with 800-grit silicon carbide abrasive paper. Subsequent mechanical polishing was carried out with 1000-grit and 1200-grit silicon carbide abrasive papers. Further smoothening of the surfaces was accomplished through polishing on MultiTex pad with alumina $\left(\mathrm{Al}_{2} \mathrm{O}_{3}\right)$ powder and distilled water. Successive polishing with decreasing sizes of alumina powder from $3.0 \mu \mathrm{m}$ to $0.1 \mu \mathrm{m}$ was carried out. The dimensions of Sample-1 and Sample- 2 after mechanical polishing are $4.2 \times 3.8 \times 1.7 \mathrm{~mm}^{3}$ and $4.0 \times 3.4 \times 1.6 \mathrm{~mm}^{3}$ respectively.

Electrical contacts are needed for some of the experiments: current-voltage (I-V) measurements, charge transport measurements, and detector response measurements. For these experiments gold electrical contacts were deposited on the opposite large sides of each sample. The electroless deposition method was used for the gold contacts by pipetting drops of $5 \%$ gold chloride $\left(\mathrm{AuCl}_{3}\right)$ solution on each planner surface. After reaction with the surface to form the contacts, excess gold chloride solution is removed from the surfaces using a felt paper to absorb the solution [9].

\subsection{Characterization Instruments and Experiments}

After mechanical polishing, the samples were thoroughly rinsed with distilled water and dried using compressed air. This is followed by characterization experiments that include XPS, current-voltage (I-V) measurements, and detector response measurements. The first set of measurements were used to collect the data on the samples before chemical etching and chemo-mechanical polishing processes.

Prior to the chemical etching and chemo-mechanical polishing, the gold contacts were removed by mechanical polishing using alumina powder from $3.0 \mu \mathrm{m}$ to $0.1 \mu \mathrm{m}$. After rinsing in distilled water and drying with compressed air, Sample 1 was chemo-mechanically polished on a special felt pad using the same chemical solution. Sample 2 was chemically etched by dipping and stirring it in a solution of hydrogen bromide in hydrogen peroxide and ethylene glycol mixture for about 2 minutes. The chemo-mechanical polishing lasted for about $1.5 \mathrm{mi}-$ nutes for each of the two large surfaces. The set of characterization experiments were then carried out for the samples.

The XPS equipment is an RHK Technology UHV 7500 system. It has an ultrahigh vacuum chamber with a pressure below $8 \times 10^{-10} \mathrm{~Pa}$. The system is equipped with an $\mathrm{Al} / \mathrm{Mg} \mathrm{X}$-ray source. We scanned for energy peaks of cadmium $(\mathrm{Cd})$, tellurium $(\mathrm{Te})$ and tellurium oxide $\left(\mathrm{TeO}_{2}\right)$.

A customized aluminum box, equipped with a Keithley picoammeter and voltage source was used for the I-V measurements. We applied voltages from -100 $\mathrm{V}$ to $100 \mathrm{~V}$ and recorded the corresponding current. A special sample holder by $\mathrm{eV}$ Products was used in the experiment for recording the response of the $\mathrm{CdZnTe}$ samples to the 59.5 gamma line of sealed ${ }^{241} \mathrm{Am}$ radiation source. The sample holder is made of brass and has a beryllium window. The sample holder is connected to a multichannel analyzer (MCA) via a pre-amplifier and an amplifier. A computer displays and record the datafrom the MCA. The ${ }^{241} \mathrm{Am}$ spec- 
tra for the two samples were recorded at an applied voltage of $100 \mathrm{~V}$.

\section{Results and Discussion}

\subsection{XPS Analysis of $\mathrm{TeO}_{2}$ on CdZnTe Surfaces}

The dominant surface species on CdZnTe wafers are cadmium (Cd), tellurium (Te) and tellurium oxide $\left(\mathrm{TeO}_{2}\right)$. This study focused on $\mathrm{Te}$ and $\mathrm{TeO}_{2}$ species. The XPS results are in Figure 1 show the $\mathrm{Te}$ and $\mathrm{TeO}_{2}$ peaks. The Te elemental states for the $3 \mathrm{~d}_{3 / 2}$ and $3 \mathrm{~d}_{5 / 2}$ doublets binding energies appear approximately at $583.5 \mathrm{eV}$ and $573.1 \mathrm{eV}$ respectively [16]. The $\mathrm{TeO}_{2} 3 \mathrm{~d}_{3 / 2}$ and $3 \mathrm{~d}_{5 / 2}$ doublets appear approximately at $586.5 \mathrm{eV}$ and $576.1 \mathrm{eV}$ respectively [16]. These binding energies were reported by Egarievwe et al. [4] with an estimated error of $\pm 0.4 \mathrm{eV}$, and by Bahl et al. [16] with and estimated error of $\pm 0.2 \mathrm{eV}$.

Figure 1(a) show that more $\mathrm{TeO}_{2}$ are formed after chemical etching as indicated by the $T e 3 d_{3 / 2} \mathrm{O}_{2}$ and $T e 3 \mathrm{~d}_{5 / 2} \mathrm{O}_{2}$ peaks. In contrast, the $\mathrm{Te} 3 \mathrm{~d}_{3 / 2} \mathrm{O}_{2}$ and Te $3 d_{5 / 2} \mathrm{O}_{2}$ peaks slightly decreased for the chemo-mechanical polishing process as shown in Figure 1(b). The importance of these results is in the effect of $\mathrm{TeO}_{2}$ on surface leakage current and noise in CdZnTe detectors.

The formation of $\mathrm{TeO}_{2}$ reduces the surface leakage current [17] [18], and hence reduces the detector noise [19] thereby improving the energy resolution of the detector [17]. The etching process using the same chemical (solution of hydrogen bromide in hydrogen peroxide and ethylene glycol mixture) resulted in the formation of more $\mathrm{TeO}_{2}$ compared to the chemo-mechanical polishing process. Figure 1(a) is from the same set of data as the XPS spectra reported in [20] where Sample-2 was used in a previous experiment as Wafer-1.

\subsection{Leakage Current}

The I-V curves for the CdZnTe wafers are shown in Figure 2. The electrical resistivity for each sample is of the order of $10^{10} \Omega-\mathrm{cm}$. The resistivity $\rho$ was calculated from the slope of the I-V curve, the thickness of the wafer $L$, and the area $A$ of the gold contacts as follow:

$$
\begin{gathered}
\rho=R(A / L) \\
R=\Delta V / \Delta I=1 / \text { Slope }
\end{gathered}
$$

where $R$ is resistance and $\Delta I / \Delta V$ is the slope.

An increase in current for each applied voltage was observed for the two wafers that a freshly processed (I-V measurement were made immediately after each process: chemicaletching and chemo-mechanical polishing). The chemo-mechanical polishing process increased the leakage current more that the chemical etching. This could be seen more clearly from Figure 3 . These results are for measurements taken immediately after processing (about 1 to 2 hours). The currents after the surface treatments usually decrease towards that of the mechanical polishing after several days [4] [21]. The measured current comes from the combination of the bulk current and the surface current. The bulk 


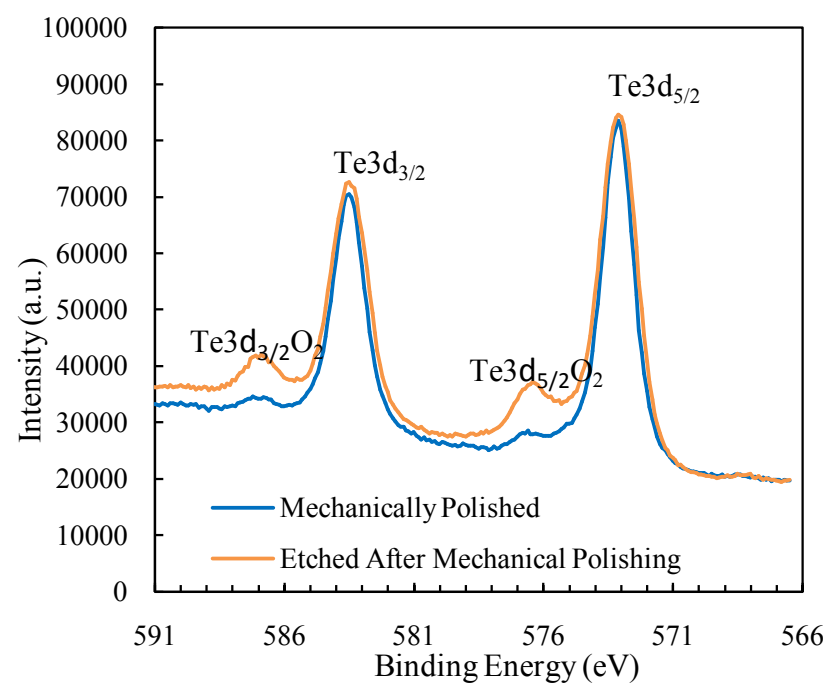

(a) Chemical etching

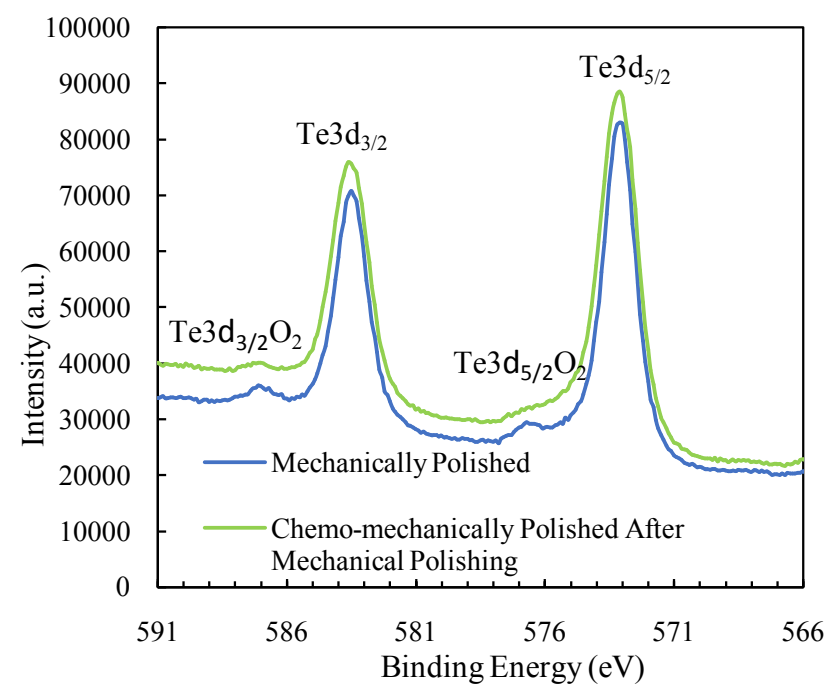

(b) Chemo-mechanical polishing

Figure 1. XPS spectra showing Te and $\mathrm{TeO}_{2}$ peaks: (a) Chemically etched after mechanical polishing (from the same data set repoted in [20]), and (b) Chemomechanically polished after mechanical polishing.

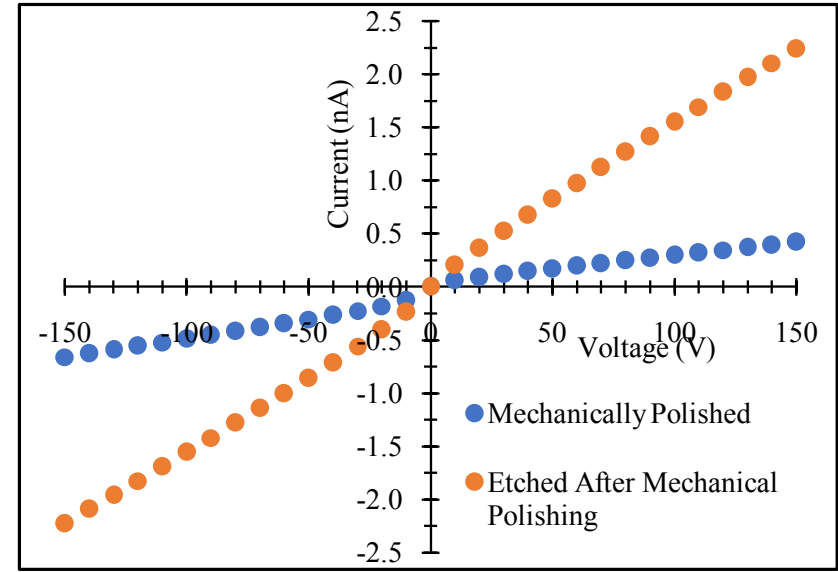

(a) Chemical etching

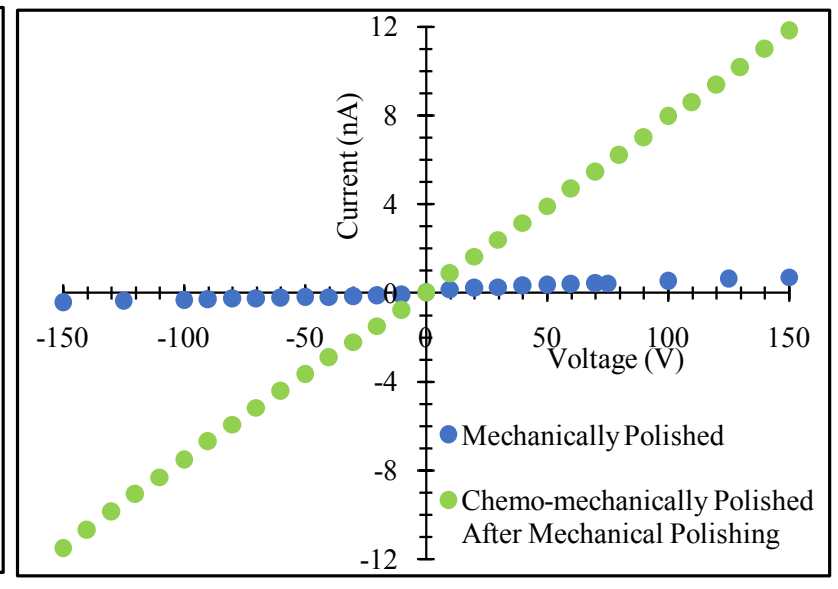

(b) Chemo-mechanical polishing

Figure 2. Current-voltage characteristics of the samples: (a) Chemically etched (from the same data set reported in [20]), and (b) Chemomechanically polished, after mechanical polishing.

current depends on the CdZnTe material. The surface current depends on the surface composition and the detector-contact interface [4]. The observed changes in the measured current come from the changes in the surface current because of the chemical treatments [4], in this case, chemical etching and chemo-mechanical polishing.

\subsection{Detector Resolution}

The spectral response measurements are shown in Figure 4 and Figure 5 for the $59.5 \mathrm{keV}$ gamma line of ${ }^{241} \mathrm{Am}$. The energy resolution immediately after chemical etching went from $21 \%$ to $32 \%$ full-width-at-half-maximum (FWHM). The FWHM immediately after chemo-mechanical polishing increased from $18 \%$ to 


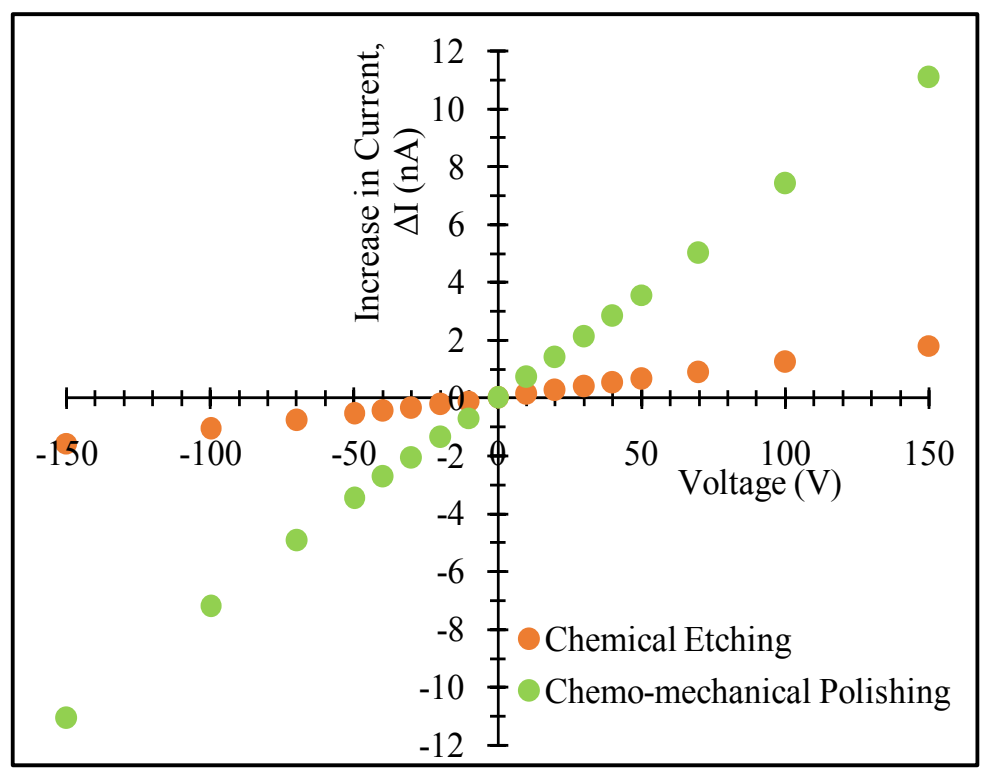

Figure 3. Increase in current, $\Delta I$, for the chemical etching (Sample-1) and chemo-mechanical polishing (Sample-2).

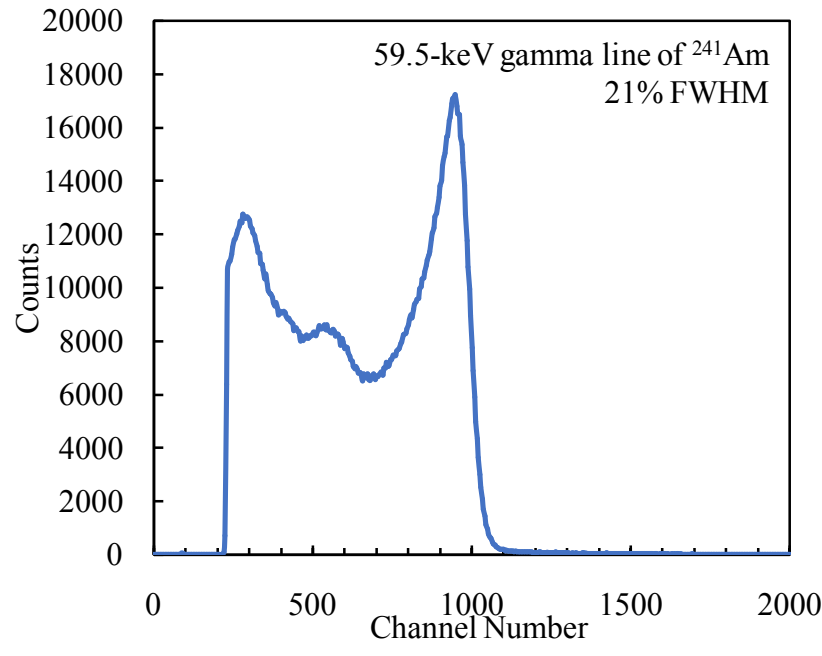

(a) Mechanically polished

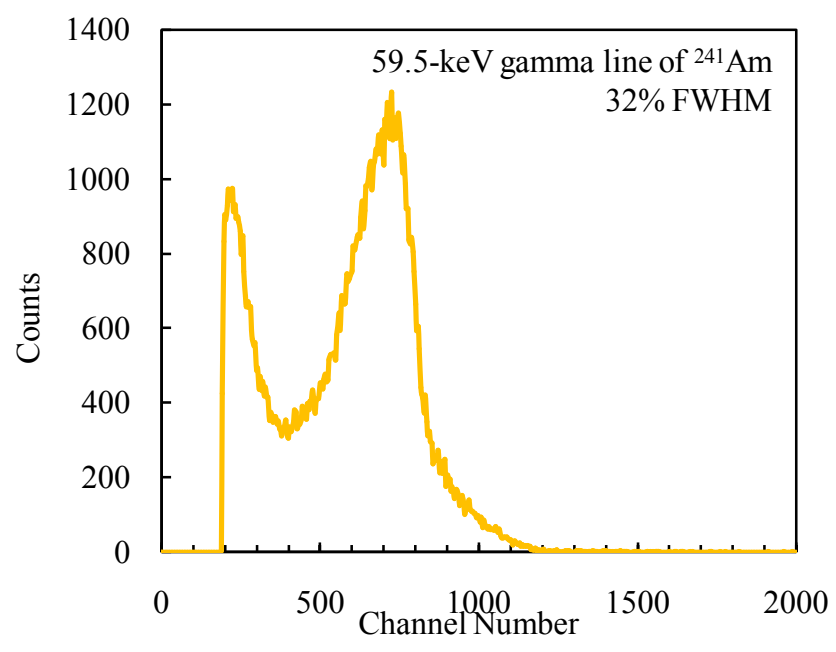

(b) Etched after mechanical polishing

Figure 4. Response of Sample-1 to the $59.5-\mathrm{keV}$ gamma line of ${ }^{241} \mathrm{Am}$ at $100 \mathrm{~V}$ : (a) mechanically polished, and (b) chemically etched after mechanical polishing (from the same data set reported in [20]).

49\%. The increase in FWHM for the freshly chemically etched sample is 52\% compared to $172 \%$ for the freshly chemo-mechanically polished sample. Thus, the etching process is more detrimental to the energy resolution than the chemo-mechanically polishing. This observation is similar to results reported for CdZnTe detectors treated with bromine-methanol-ethylene glycol [12]. The reduction in energy resolution agrees with the increase in surface current which causes increase in detector noise. Increase in detector noise lowers the energy resolution. It is expected that the energy resolutions will improve as the treated surfaces stabilize and the measured leakage currents reduces. 


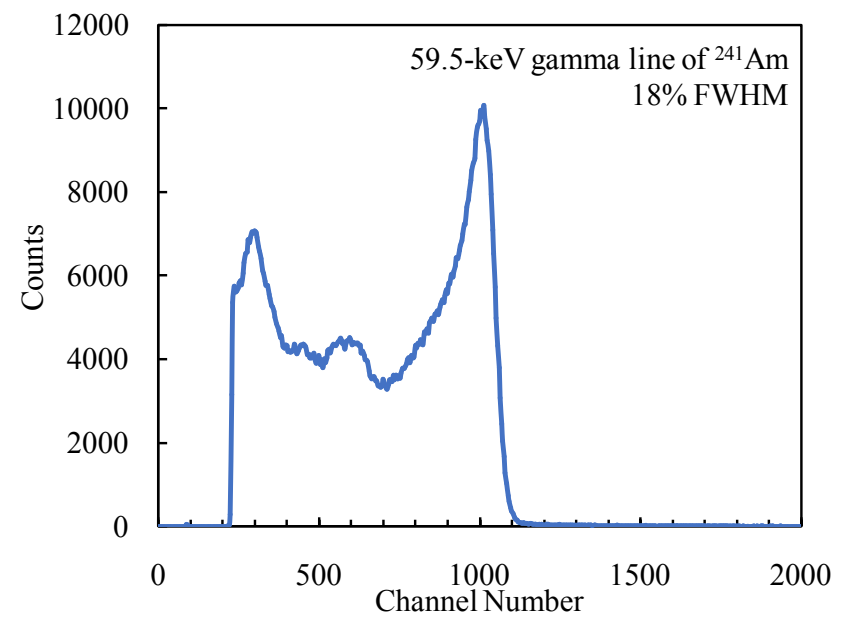

(a) Mechanically polished

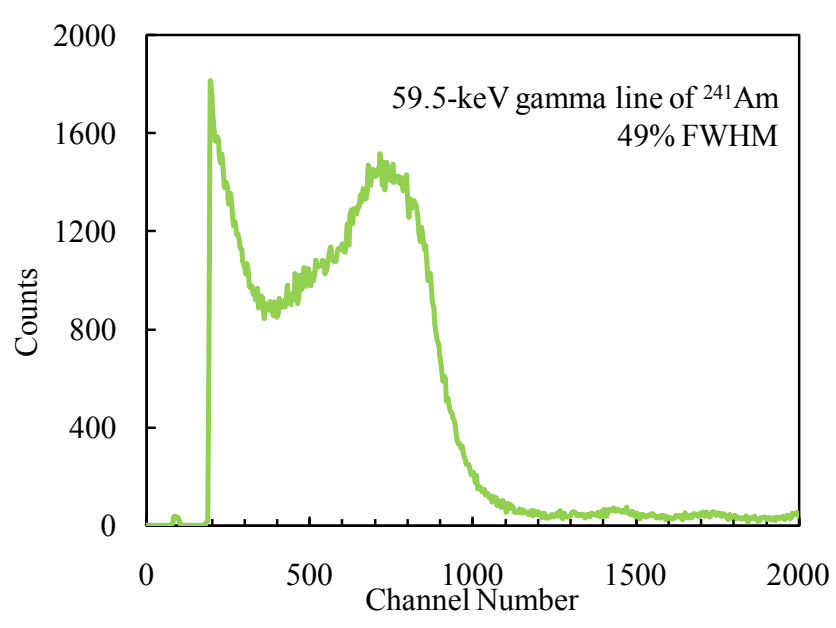

(b) Etched after mechanical polishing

Figure 5. Response of Sample-2 to the 59.5-keV gamma line of ${ }^{241} \mathrm{Am}$ at $100 \mathrm{~V}$ : (a) Mechanically polished, and (b) Chemo-mechanically polished after mechanical polishing.

\section{Conclusions}

The effect of etching and chemo-mechanical polishing processes on CdZnTe nuclear detectors was studied using the same chemical solution (hydrogen bromide in hydrogen peroxide and ethylene glycol mixture). The XPS experiments showed that the chemical etching process resulted in the formation of more $\mathrm{TeO}_{2}$ on the detector surfaces compared to chemo-mechanical polishing. The electrical resistivity of the detector, obtained from the slope of the I-V curve, the wafer thickness, and the gold contact area is of the order of $10^{10} \Omega-\mathrm{cm}$. It was observed that the chemo-mechanical polishing process increased the leakage current more that chemical etching.

The etching process, for freshly treated surfaces, is more detrimental to the energy resolution than chemo-mechanically polishing. The currents after the surface treatments are expected to decrease towards that of the mechanical polishing after several days [4] [21]. This should lead to improved energy resolutions of the CdZnTe wafers. Further studies on aging of the treated samples for longer periods are needed to understand the long-term effects of these processes.

\section{Acknowledgements}

This research was funded by the US Department of Homeland Security, Domestic Nuclear Detection Office, under competitively awarded contract/IAA award number 2012-DN-077-ARI065-05; the US Department of Energy, Office of Defense Nuclear Nonproliferation Research and Development, DNN R\&D (NA-22); the US Nuclear Regulatory Commission through award number NRC-27-10-514; and the National Science Foundation (NSF) HBCU-UP Program through award number 1818732 .

\section{Conflicts of Interest}

The authors declare no conflicts of interest regarding the publication of this paper. 


\section{References}

[1] Egarievwe, S.U., Chan, W., Kim, K.H., Roy, U.N., Sams, V., Hossain, A., Kassu, A. and James, R.B. (2016) Carbon Coating and Defects in CdZnTe and CdMnTe Nuclear Detectors. IEEE Transactions on Nuclear Science, 63, 236-245. https://doi.org/10.1109/TNS.2016.2515108

[2] Limousin, O. (2003) New Trends in CdTe and CdZnTe Detectors for X- and Gamma-Ray Applications. Nuclear Instruments and Methods in Physics Research Section $A, 504,24-37$.

[3] Yadav, J.S., Savitri, S. and Malkar, J.P. (2005) Near Room Temperature X-Ray and $\gamma$-Ray Spectroscopic Detectors for Future Space Experiments. Nuclear Instruments and Methods in Physics Research Section A, 552, 399-408. https://doi.org/10.1016/j.nima.2005.07.001

[4] Egarievwe, S.U., Hossain, A., Okwechime, I.O., Egarievwe, A.A., Jones, D.E., Roy, U.N. and James, R.B. (2016) Effects of Chemical Treatments on CdZnTe X-Ray and Gamma-Ray Detectors.IEEE Transactions on Nuclear Science, 63, 1091-1098. https://doi.org/10.1109/TNS.2016.2527779

[5] James, R.B., Schlesinger, T.E., Lund, J.C. and Schieber, M. (1995) Semiconductors for Room Temperature Nuclear Detector Applications. Academic Press, San Diego.

[6] Zhang, Q., Zhang, C., Lu, Y., Yang, K. and Ren, Q. (2013). Progress in the Development of CdZnTe Unipolar Detectors for Different Anode Geometries and Data Corrections. Sensors, 13, 2447-2474. https://doi.org/10.3390/s130202447

[7] Barber, H.B. (1999). Applications of Semiconductor Detectors to Nuclear Medicine. Nuclear Instruments and Methods in Physics Research Section A: Accelerators, Spectrometers, Detectors and Associated Equipment, 436, 102-110. https://doi.org/10.1016/S0168-9002(99)00605-1

[8] Verger, L., Boitel, M., Gentet, M.C., Hamelin, R., Mestais, C., Mongellaz, F., Rustique, J. and Sanchez, G. (2001) Characterization of CdTe and CdZnTe Detectors for Gamma-Ray Imaging Applications. Nuclear Instruments and Methods in Physics Research Section A: Accelerators, Spectrometers, Detectors and Associated Equipment, 458, 297-309. https://doi.org/10.1016/S0168-9002(00)00874-3

[9] Egarievwe, S.U., Hossain, A., Okwechime, I.O., Gul, R. and James, R.B. (2015) Effects of Chemomechanical Polishing on CdZnTe X-Ray and Gamma-Ray Detectors. Journal of Electronic Materials, 44, 3194-3201. https://doi.org/10.1007/s11664-015-3881-7

[10] Hossain, A., Dowdy, A., Bolotnikov, A.E., Camarda, G.S., Cui, Y., Roy, U.N., Tappero, R., Tong, X., Yang, G. and James, R.B. (2014) Topographic Evaluation of the Effect of Passivation in Improving the Performance of CdZnTe Detectors. Journal of Electronic Materials, 43, 2941-2946. https://doi.org/10.1007/s11664-014-3153-y

[11] Drabo, M.L., Egarievwe, S.U., Okwechime, I.O., Jones, D.E., Hossain, A. and James, R.B. (2017) Analysis of $\mathrm{Te}$ and $\mathrm{TeO}_{2}$ on CdZnTe Nuclear Detectors Treated with Hydrogen Bromide and Ammonium-Based Solutions. Journal of Materials Science and Chemical Engineering, 5, 9-18. https://doi.org/10.4236/msce.2017.54002

[12] Egarievwe, S.U., Jow, J.O., Egarievwe, A.A., Gul, R., Martin, R.D., Hales, Z.M., Hossain, A., Roy, U.N. and James, R.B. (2015) Effects of Etching and Chemo-Mechanical Polishing on the Electrical Properties of CdZnTe Nuclear Detectors. American Journal of Materials Science, 5, 16-20.

[13] Fougeres, P., Hage-Ali, M., Koebel, J.M., Siffert, P., Hassan, S., Lusson, A., Triboulet, R., Marrakchi, A. Zerrai, A., Cherkaoui, K., Adhiri, R., Bremond, G., Kaitasovd, O., Ruaultd, M.O. and Crestoue, J. (1998) Properties of $\mathrm{Cd}_{1-\mathrm{x}} \mathrm{Zn}_{\mathrm{x}}$ Te Crystals Grown 
by High Pressure Bridgman for Nuclear Detection. Journal of Crystal Growth, 184, 1313-1318. https://doi.org/10.1016/S0022-0248(98)80271-6

[14] Doty, F.P., Butler, J.F., Schetzina, J.F. and Bowers, K.A. (1992) Properties of CdZnTe Crystals Grown by a High Pressure Bridgman Method. Journal of Vacuum Science \& Technology B, 10, 1418-1422. https://doi.org/10.1116/1.586264

[15] Kim, K.H., Bolotnikov, A.E., Camarda, G.S., Tappero, R., Hossain, A., Cui, Y., Franc, J., Marchini, L., Zappettini, A., Fochuk, P., Yang, G., Gul, R. and James, R.B. (2012) New Approaches for Making Large-Volume and Uniform CdZnTe and CdMnTe Detectors. IEEE Transactions on Nuclear Science, 59, 1510-1515. https://doi.org/10.1109/TNS.2012.2202917

[16] Bahl, M.K., Watson, R.L. and Irgolic, K.J. (1977) X-Ray Photoemission Studies of Tellurium and Some of Its Compounds. The Journal of Chemical Physics, 66, 5526-5535. https://doi.org/10.1063/1.433874

[17] Wright, G.W., James, R.B., Burger, A. and Chinn, D.A. (2003) U.S. Patent No. 6649915. U.S. Patent and Trademark Office, Washington DC.

[18] Özsan, M.E., Sellin, P.J., Veeramani, P., Hinder, S.J., Monnier, M.L.T., Prekas, G., Lohstroh, A. and Baker, M.A. (2010) Chemical Etching and Surface Oxidation Studies of Cadmium Zinc Telluride Radiation Detectors. Surface and Interface Analysis, 42, 795-798. https://doi.org/10.1002/sia.3146

[19] Chattopadhyay, K., Hayes, M., Ndap, J.O., Burger, A., Lu, W.J., McWhinney, H.G., Grady, T. and James, R.B. (2000) Surface Passivation of Cadmium Zinc Telluride Radiation Detectors by Potassium Hydroxide Solution. Journal of Electronic Materials, 29, 708-712. https://doi.org/10.1007/s11664-000-0210-5

[20] Egarievwe, S.U., Lukosi, E.D., Okwechime, I.O., Gul, R., Hossain, A. and James, R.B. (2017) Effects of Tellurium Oxide on Surface Current and Performance of CdZnTe Nuclear Radiation Detectors. 2017 IEEE Nuclear Science Symposium and Medical Imaging Conference (NSS/ MIC), Atlanta, GA, 21-28 October 2017, 1-4. https://doi.org/10.1109/NSSMIC.2017.8532704

[21] Okwechime, I.O., Egarievwe, S.U., Hossain, A., Hales, Z.M., Egarievwe, A.A. and James, R.B. (2014) Chemical Treatment of CdZnTe Radiation Detectors Using Hydrogen Bromide and Ammonium-Based Solutions. Proceedings of Hard X-Ray, Gamma-Ray, and Neutron Detector Physics XVI, 92130Y.

https://doi.org/10.1117/12.2063067 